\title{
Evaluación del desplazamiento del sobreengrasante en jabones blandos.
}

\author{
Por N. Crespo González y D. Prada Rodríguez* \\ Departamento de Química Analítica. Facultad de Ciencias. \\ Universidad de La Coruna. Campus de Zapateira. 15071. La Coruña.
}

RESUMEN

Evaluación del desplazamiento del sobreengrasante en jabones blandos.

Se estudia, comprueba y cuantifica el fenómeno de la metátesis que ocurre en jabones reengrasados con ácidos grasos. El análisis se refiere a jabones en disolución (cremas de afeitar) en los cuales el problema es más patente debido a fenómenos de hidrólisis. El estudio se centra en los ácidos grasos mayoritarios de las muestras analizadas. Conociendo la distribución de los ácidos grasos se estimaron las concentraciones que teóricamente debían ser encontradas y fueron comparadas con las concentraciones halladas por cromatografia en fase gaseosa.

PALABRAS-CLAVE: Acido graso - Jabón blando - Metátesis - Reengrasado - Sobreengrasante.

\section{SUMMARY}

Evaluation of the displacement of the superfatting agent in soft soaps.

The metathesis that occurs in soaps superfatted with fatty acids is studied, verified and evaluated. The analysis deals with soaps in solution (shaving creams), in which the problem is showed clearly due to the hidrolysis of the soap. The study is concern on the main fatty acids of the analized samples. Knowing the fatty acids distribution, the axpected theoretical concentrations of the fatty acids were estimated and were compared with those found by Gas Chromatography.

KEY-WORDS: Fatty acid - Metathesis - Soft soap - Superfatted Superfatting agent.

\section{INTRODUCCION}

Un alto porcentaje del jabón que se hace actualmente contiene sobreengrasante. La cantidad de éste que se añade puede variar entre un $0,5 \%$ y un $10 \%$. El tipo y naturaleza de este tipo de aditivos es prácticamente ilimitada, aunque los sobreengrasantes más frecuentemente utilizados incluyen lanolinas y derivados de las mismas, o simplemente la cantidad suficiente de ácidos grasos capaces de neutralizar la pequeña cantidad de álcali residual, y permanecer en un cierto exceso, en un jabón base común.
Los ácidos grasos más frecuentemente utilizados con este fin son los ácidos grasos destilados de palma, de almendra, de coco y ácido esteárico de alta calidad (1).

Los jabones que contienen ácidos grasos como sobreengrasante presentan un interesante problema para el químico que pretende realizar un análisis completo de una muestra desconocida, y en particular si pretende conocer la naturaleza de los ácidos grasos libres.

El método normal para la extracción de estos ácidos grasos es disolver la muestra de jabón en agua y realizar una extracción líquido-líquido con éter de petróleo o éter etílico. Los extractos combinados se secan sobre sulfato sódico, se evaporan a sequedad, y se examinan los ácidos grasos resultantes por cromatografía en fase gaseosa, tras la conversión en los correspondientes ésteres metíli$\cos (2)(3)(4)(5)$.

EI resultado de este tipo de análisis es interesante, especialmente en lo que se refiere a la naturaleza de los ácidos grasos extraídos. Si, por ejemplo, el método se ha utilizado para identificar un $10 \%$ de ácido esteárico que ha sido añadido a un jabón comercial de sebo-aceite de coco en proporción 80:20, se encuentra que el extracto, en todos los casos se parece a la mezcla original 80:20, $y$, por lo tanto, difiere mucho del ácido graso que se pensaba encontrar. Resultados similares se obtienen con otros ácidos grasos utilizados como reengrasantes en mezclas de distintas proporciones de grasa (6). Este fenómeno fue denominado METATESIS, atendiendo a la definición común que entiende como metátesis la reacción química en la cual se produce un intercambio de elementos, iones o radicales de acuerdo con la ecuación:

$$
\mathrm{A}^{+} \mathrm{B}^{+}+\mathrm{C}^{+} \mathrm{D}^{-}=\mathrm{A}^{+} \mathrm{D}^{+}+\mathrm{C}^{+} \mathrm{B}^{-}
$$

Estos resultados parecen sugerir que cierto tipo de metátesis ha tenido lugar en el jabón en estado líquido o incluso en el jabón disuelto en agua, quizás debido a la parcial hidrólisis del mismo; sin embargo, en la bibliografía se han descrito estudios con jabones preparados en el laboratorio por mezcla sólida del reengrasante o incluso por extracción en soxhlet en atmósfera anhidra, obteniéndose resultados similares (7). 
Este trabajo tiene por objeto evaluar hasta qué punto la metátesis se pone de manifiesto en jabones mantenidos en disolución, como es el caso de las cremas de afeitar, estudiando qué tipos de ácidos grasos se intercambian preferentemente en las distintas formulaciones, así como observar la influencia de las condiciones de temperatura a que pueden estar sometidos estos jabones, para conocer cual es la composición del jabón que el usuario encuentra a la hora de utilizarlo.

Para realizar este estudio se ha partido de tres cremas de afeitar de tipo comercial distintas, de las cuales se conocía previamente su composición.

Todas las cremas fueron sometidas durante tres meses a distintas condiciones de temperatura dentro de su envase. Transcurridos este tiempo fueron analizadas.

Las características principales de las cremas analizadas se detallan en la Tabla I, donde se puede comprobar que tanto la cantidad de ácidos grasos saponificados, como la cantidad de ácidos grasos libres (no saponificados) es distinta en todas ellas.

Tabla I

Características más importantes de las cremas estudiadas.

\begin{tabular}{lrr|r|} 
& CREMA I & CREMA II & CREMA III \\
\hline \% Ac. Gr. Libres & 3,2 & 1,7 & 4,7 \\
\% Ac. Gr. Saponificados & 49,0 & 41,0 & 39,0 \\
Relación Na:K & $1: 5,9$ & $1: 8$ & $1: 9,2$ \\
\% Agua & 34,0 & 38,0 & 40,0 \\
\% Glicerina & 6,5 & 9,3 & 12,4 \\
Conc. Glicerina \% & 16,0 & 19,7 & 23,5 \\
Conc. jabón Na:K & 55,4 & 46,4 & 44,7 \\
\hline
\end{tabular}

Los dos primeros jabones (I y II) se componen de una base de estearina (ácido esteárico)-coco 70:30, mientras que la composición del tercer jabón supone una mezcla grasa distinta estearina-coco-cacahuete 70:20:10. En todos los casos el sobreengrasante empleado fue la estearina, en cantidad suficiente como para saponificar el álcali libre residual y quedar un pequeño resto como sobreengrasante.

\section{PARTE EXPERIMENTAL}

Se estudiaron los efectos de una posible metátesis en tres formulaciones de crema de afeitar distintas, cuya concentración en ácidos grasos teóricos era la siguiente:

\section{CREMA I: SAPONIFICADOS}

Coco: $\quad 10,08 \%$

Estearina: $\quad 38,92 \%$ LIBRES

Estearina: $3,18 \%$

CREMA II: SAPONIFICADOS

Coco: $\quad 8,81 \%$

Estearina: $32,19 \%$

\section{LIBRES}

Estearina: $1,68 \%$

\section{CREMA III: SAPONIFICADOS}

Cacahuete: $2,45 \%$

Coco: $\quad 5,96 \%$

Estearina: $30,59 \%$

LIBRES

$$
\text { Estearina: } 4,67 \%
$$

Se hicieron cromatogramas de las grasas empleadas para conocer su composición en ácidos grasos, ya que interesa conocer cómo se comporta cada ácido graso en cada una de las muestras estudiadas. Para este estudio se han considerado los ácidos grasos mayoritarios de estas mezclas grasas, por lo que los cálculos se han referido, en todos los casos, a ácido laúrico $\left(\mathrm{C}_{12}\right)$, ácido mirístico $\left(\mathrm{C}_{14}\right)$, ácido palmítico $\left(\mathrm{C}_{16}\right)$, ácido esteárico $\left(\mathrm{C}_{18}\right)$, ácido oleico $\left(\mathrm{C}_{18}\right)$ y ácido linoleico $\left(\mathrm{C}_{18=}\right)$. En la Tabla II se detallan las composiciones encontradas para cada una de las grasas que constituyen las cremas de afeitar, así como la estearina utilizada como sobreengrasante.

Tabla II

Composición, en \% de ácidos grasos mayoritarios, de las grasas que constituyen las cremas de afeitar.

\begin{tabular}{|l|c|c|c|}
\hline Coco & Estearina & Cacahuete \\
\hline$C_{12}$ & $48 \%$ & $0,7 \%$ & \\
\hline$C_{14}$ & $17 \%$ & $2,3 \%$ & \\
\hline$C_{18}$ & $9 \%$ & $52,3 \%$ & $14,6 \%$ \\
\hline$C_{18}$ & $2 \%$ & $44,6 \%$ & $3,7 \%$ \\
\hline$C_{18}-$ & $7 \%$ & & $42,2 \%$ \\
\hline$C_{18}$ & $2 \%$ & & $39,5 \%$ \\
\hline
\end{tabular}

Todas las cremas fueron mantenidas a $-20^{\circ} \mathrm{C}$, temperatura ambiente y $45^{\circ} \mathrm{C}$ durante tres meses, al término de los cuales se realizaron los análisis.

Los análisis consistieron en la extracción de los ácidos grasos libres y posterior separación de los ácidos grasos saponificados de la fase acuosa; conversión en los ésteres metílicos correspondientes y análisis por cromatografía en fase gaseosa. El método analítico empleado fue el siguiente:

\section{EXTRACCION DE ACIDOS GRASOS LIBRES:}

Se realiza una extracción con éter etílico del jabón disuelto en agua. Se lavan las fases etéreas con agua, se secan sobre sulfato sódico y se concentran a sequedad.

\section{EXTRACCION DE LOS ACIDOS GRASOS SAPONIFI- CADOS:}

Las fases acuosas resultantes se acidulan con $\mathrm{HCl}$ concentrado hasta completa hidrólisis del jabón. Se extrae la grasa con éter etílico, se lava la fase etérea con agua, se seca sobre sulfato sódico y se concentra a sequedad. 
PREPARACION DE LOS ESTERES METILICOS:

Se prepararon los ésteres metílicos correspondientes a los ácidos grasos siguiendo la norma A.O.A.C. 969-33 (8).

\section{CONDICIONES CROMATOGRAFICAS:}

Se utilizó un cromatógrafo Perkin Elmer, modelo 8310 , equipado con una sola columna y detector de ionización de llama, con compensación automática de la línea de base para trabajos a temperatura programada. La columna utilizada es de acero inoxidable de $2 \mathrm{~m} \times 1 / 8$ " con $10 \%$ de EGSS-X sobre Chromosorb W.HP, $80 / 100$ mesh. (9).

La temperatura del horno fue, inicialmente, de $135^{\circ} \mathrm{C}$, programándose, a $4^{\circ} \mathrm{C} / \mathrm{min}$. a partir del segundo minuto de efectuada la inyección, hasta alcanzar los $198^{\circ} \mathrm{C}$. EI flujo del gas portador (nitrógeno) fue de $30 \mathrm{~mL} / \mathrm{min}$.

Se utilizó un patrón de ácidos grasos conteniendo concentraciones conocidas de $\mathrm{C}_{12}, \mathrm{C}_{14}, \mathrm{C}_{16}, \mathrm{C}_{18}, \mathrm{C}_{18 .}$ y $\mathrm{C}_{18}$ de Supelco S.A. (10).

\section{RESULTADOS Y DISCUSION}

Estudiando por cromatografía de gases (C.G.) los ácidos grasos libres de las cremas se encuentra que la acidez libre en todas ellas contiene ácidos grasos no aportados por el ácido esteárico (fundamentalmente $\mathrm{C}_{16}$ y $\mathrm{C}_{18}$ ) añadido como sobreengrasante para mejorar la calidad de la espuma y proporcionar el típico brillo perlado. Tabla III

Tabla III

Composición del insaponificado a temperatura ambiente (valores expresados en $\%$ de milimoles)

\begin{tabular}{|c|c|c|c|}
\hline TEORICO & I & II & III \\
\hline $\begin{array}{l}\mathrm{C}_{12} \\
\mathrm{C}_{14} \\
\mathrm{C}_{18} \\
\mathrm{C}_{18} \\
\mathrm{C}_{18-} \\
\mathrm{C}_{18}= \\
\end{array}$ & $\begin{array}{r}0.98 \\
2.72 \\
54.49 \\
41.81 \\
0.00 \\
0.00 \\
\end{array}$ & $\begin{array}{r}0.97 \\
2.64 \\
54.74 \\
41.64 \\
0.00 \\
0.00 \\
\end{array}$ & $\begin{array}{r}0.99 \\
3.23 \\
54.15 \\
41.63 \\
0.00 \\
0.00 \\
\end{array}$ \\
\hline HALLADO POR G.C. & I & II & I I I \\
\hline $\begin{array}{l}C_{12} \\
C_{14} \\
C_{18} \\
C_{18} \\
C_{18-} \\
C_{18}= \\
\end{array}$ & $\begin{array}{r}7.42 \\
6.20 \\
48.08 \\
38.30 \\
0.00 \\
0.00 \\
\end{array}$ & $\begin{array}{r}9.73 \\
8.52 \\
43.19 \\
38.56 \\
0.00 \\
0.00 \\
\end{array}$ & $\begin{array}{r}4.18 \\
4.12 \\
44.86 \\
39.73 \\
5.72 \\
1.40 \\
\end{array}$ \\
\hline DIFERECIA & I & II & II I \\
\hline $\begin{array}{l}C_{12} \\
C_{14} \\
C_{18} \\
C_{18} \\
C_{18-} \\
C_{18}=\end{array}$ & $\begin{array}{r}6.44 \\
3.48 \\
-6.41 \\
-3.51 \\
0.00 \\
0.00\end{array}$ & $\begin{array}{r}8.76 \\
5.88 \\
-11.55 \\
-3.08 \\
0.00 \\
0.00\end{array}$ & $\begin{array}{r}3.19 \\
0.89 \\
-9.29 \\
-1.90 \\
5.72 \\
1.40\end{array}$ \\
\hline
\end{tabular}

Para comprobar una posible metátesis entre los ácidos grasos libres y los combinados en forma de jabón (Na-K) se determinaron éstos por C.G., encontrando que la distribución de ácidos grasos libres obtenida de los cromatogramas difería notablemente de la composición esperada para el ácido esteárico y se parecía a la composición de la mezcla grasa saponificada.
Del estudio de los valores de las Tablas III y IV se deduce que, efectivamente, tiene lugar un proceso de metátesis en el que los ácidos grasos libres añadidos $\left(C_{16}\right.$ y $\left.C_{18}\right)$ desplazan a otros de cadena más corta $\left(C_{12}\right.$ y $C_{14}$, o insaturados $C_{18 \text {. }}$ y $C_{18 z}$ ) de sus jabones hasta alcanzar un equilibrio. Esta metátesis no parece verse muy afectada en su magnitud por la temperatura a que ha estado sometida la crema, al menos en las tres temperaturas estudiadas, que cubren un amplio intervalo, $y$, sobre todo, teniendo en cuenta los errores que se pueden introducir en el largo proceso analítico. Tabla V.

\section{Tabla IV}

Composición del saponificado a Temperatura ambiente (valores expresados en $\%$ de milimoles)

\begin{tabular}{|c|c|c|c|}
\hline TEORICO & I & II & III \\
\hline $\begin{array}{l}\mathrm{C}_{12} \\
\mathrm{C}_{14} \\
\mathrm{C}_{16} \\
\mathrm{C}_{18} \\
\mathrm{C}_{18-} \\
\mathrm{C}_{18=} \\
\end{array}$ & $\begin{array}{r}5.12 \\
2.58 \\
20.39 \\
16.59 \\
0.00 \\
0.00 \\
\end{array}$ & $\begin{array}{r}4.46 \\
2.20 \\
16.72 \\
13.57 \\
0.00 \\
0.00 \\
\end{array}$ & $\begin{array}{r}3.06 \\
1.65 \\
15.14 \\
13.22 \\
4.77 \\
3.53 \\
\end{array}$ \\
\hline HALLADO POR G.C. & I & II & III \\
\hline $\begin{array}{l}\mathrm{C}_{12} \\
\mathrm{C}_{14} \\
\mathrm{C}_{18} \\
\mathrm{C}_{18} \\
\mathrm{C}_{18-} \\
\mathrm{C}_{28}= \\
\end{array}$ & $\begin{array}{r}4.98 \\
2.51 \\
20.62 \\
16.74 \\
0.00 \\
0.00 \\
\end{array}$ & $\begin{array}{r}4.31 \\
2.08 \\
16.81 \\
13.52 \\
0.00 \\
0.00 \\
\end{array}$ & $\begin{array}{r}2.96 \\
1.65 \\
16.11 \\
13.81 \\
0.00 \\
0.00 \\
\end{array}$ \\
\hline DIFERENCIA & I & II & II \\
\hline $\begin{array}{l}\mathrm{C}_{12} \\
\mathrm{C}_{14} \\
\mathrm{C}_{16} \\
\mathrm{C}_{18} \\
\mathrm{C}_{18}- \\
\mathrm{C}_{18}=\end{array}$ & $\begin{array}{r}-0.14 \\
-0.07 \\
0.23 \\
0.15 \\
0.00 \\
0.00\end{array}$ & $\begin{array}{r}-0.15 \\
-0.12 \\
0.09 \\
0.05 \\
0.00 \\
0.00\end{array}$ & $\begin{array}{r}-0.10 \\
0.00 \\
0.97 \\
0.59 \\
-3.68 \\
-2.61\end{array}$ \\
\hline
\end{tabular}

Tabla V

Diferencias de ácidos grasos libres encontrados a distintas temperaturas. Valores expresados en $\%$ de moles.

\begin{tabular}{|l|r|r|r|r|r|l|}
\hline & $\mathrm{C}_{12}$ & $\mathrm{C}_{14}$ & $\mathrm{C}_{18}$ & $\mathrm{C}_{18}$ & $\mathrm{C}_{18}-$ & $\mathrm{C}_{18}$ \\
\hline CREMA I & & & & & & \\
$-20^{\circ} \mathrm{C}$ & 7.50 & 3.67 & -8.78 & -2.39 & 0.00 & 0.00 \\
T.Amb. & 6.44 & 3.48 & -6.41 & -3.51 & 0.00 & 0.00 \\
$45^{\circ} \mathrm{C}$ & 4.81 & 2.73 & -4.44 & -3.10 & 0.00 & 0.00 \\
CREMA II & & & & & & \\
$-20^{\circ} \mathrm{C}$ & 7.65 & 4.09 & -7.70 & -4.05 & 0.00 & 0.00 \\
T. Amb. & 8.76 & 5.88 & -11.55 & -3.08 & 0.00 & 0.00 \\
$45^{\circ} \mathrm{C}$ & 10.01 & 2.67 & -8.89 & -3.88 & 0.00 & 0.00 \\
CREMA II I & & & & & & \\
-20 & & & & & \\
T.Amb. & 6.11 & 2.58 & -9.96 & -6.36 & 6.01 & 1.61 \\
$45^{\circ} \mathrm{C}$ & 3.19 & 0.89 & -9.29 & -1.90 & 5.72 & 1.40 \\
& 4.54 & 1.38 & -8.05 & -4.63 & 5.22 & 1.53 \\
\hline
\end{tabular}

La metátesis que se pone de manifiesto tampoco parece verse muy afectada por la cantidad de estearina añadida diferente en las tres cremas estudiadas. Tabla VI.

Según los datos de la Tabla VI parece establecerse un equilibrio en el desplazamiento hasta alcanzar valores semejantes de $\mathrm{C}_{12}+\mathrm{C}_{14}$, independientemente de la estearina añadida. Por otro lado, e intentando evaluar la metátesis de forma absoluta, podríamos pensar que su efecto es menor en la crema III, ya que esta parte de valores teóricos mayores, aunque no hay que olvidar que en este caso se ve aumentada por la aparición de ácidos grasos libres $C_{18 \text {. }}$ y $C_{18=}$ que teóricamente no debieran estar pre- 
Tabla VI

\begin{tabular}{|c|c|c|c|}
\hline TEORICO & CREMA I & CREMA II & CREMA III \\
\hline 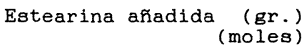 & $\begin{array}{r}3.13 \\
11.75\end{array}$ & $\begin{array}{l}1.92 \\
7.18\end{array}$ & $\begin{array}{r}5.35 \\
20.13\end{array}$ \\
\hline$\left(\mathrm{C}_{12}+\mathrm{C}_{14}\right)_{\left(\begin{array}{c}(\mathrm{gr} .) \\
\text { (moles) }\end{array}\right.}^{(}$ & $\begin{array}{l}0.096 \\
0.435\end{array}$ & $\begin{array}{l}0.058 \\
0.260\end{array}$ & $\begin{array}{l}0.165 \\
0.850\end{array}$ \\
\hline HALLADO POR G.C. & CREMA I & CREMA II & CREMA III \\
\hline$\left(\mathrm{C}_{12}+\mathrm{C}_{14}\right) \underset{\text { (moles) }}{(\text { gr. })}$ & $\begin{array}{l}0.33 \\
1.56\end{array}$ & $\begin{array}{l}0.27 \\
1.50\end{array}$ & $\begin{array}{l}0.27 \\
1.40\end{array}$ \\
\hline DIFERENCIA & CREMA I & CREMA II & CREMA III \\
\hline$\left(\mathrm{C}_{12}+\mathrm{C}_{14}\right)_{\left(\begin{array}{c}\text { (gr. }) \\
\text { (moles) }\end{array}\right.}$ & $\begin{array}{l}0.234 \\
1.125\end{array}$ & $\begin{array}{l}0.212 \\
1.240\end{array}$ & $\begin{array}{l}0.105 \\
0.550\end{array}$ \\
\hline
\end{tabular}

sentes y en los que se observa que son preferentemente desplazados.

En las Figuras 1-3 donde se muestran los perfiles de los ácidos grasos teóricos y los calculados, se pone de manifiesto el desplazamiento del sobreengrasante estudiado, considerando los valores teóricos que debían ser encontrados.

\section{METATESIS EN LA CREMA I A TEMPERATURA AMBIENTE}

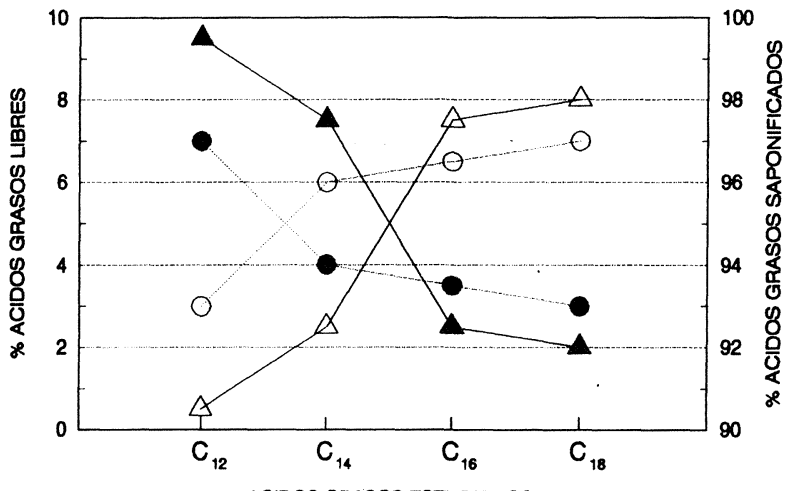

ACIDOS GRASOS ESTUDIADOS

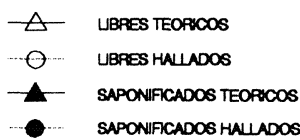

Figuras 1

Comparación entre los ácidos grasos teóricos y los hallados en la crema I a temperatura ambiente.

En las Figuras 4-6 se comprueba que la distribución de los ácidos grasos libres hallados es similar a.la de los ácidos grasos saponificados, $y$, por tanto, difiere notablemente de la distribución esperada para el reengrasante añadido.

Esta metátesis tiene como consecuencia un cierto enriquecimiento de la crema en jabones palmíticos y esteáricos, menos solubles, en detrimento de los laúricos o insaturados de mayor facilidad espumante en frío.

El fenómeno había sido ocasionalmente estudiado en los jabones de tocador sobreengrasados con ácidos grasos en presencia de agua, apuntándose que la acidez
METATESIS'EN LA CREMA II A TEMPERATURA AMBIENTE

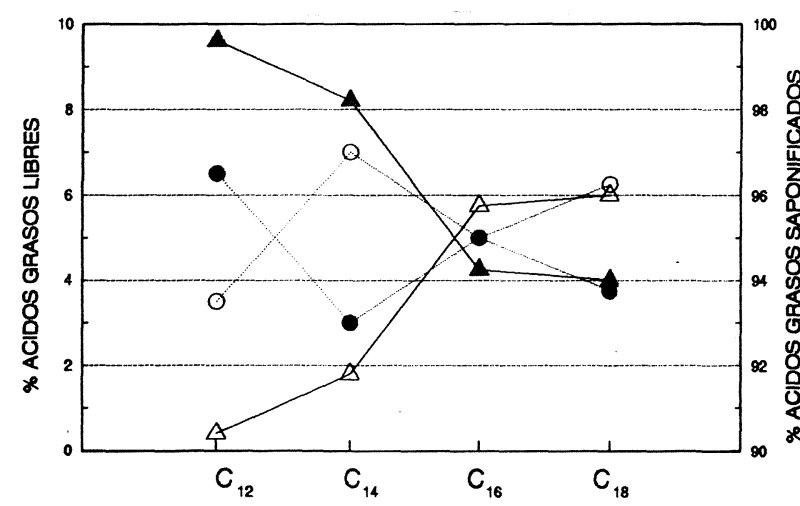

ACIDOS GRASOS ESTUDIADOS

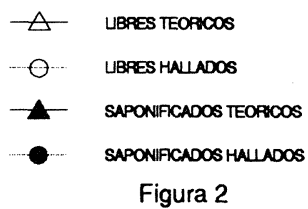

Figura 2

Comparación entre los ácidos grasos teóricos y los hallados en la crema II a temperatura ambiente.

\section{METATESIS EN LA CREMA III A TEMPERATURA AMBIENTE}

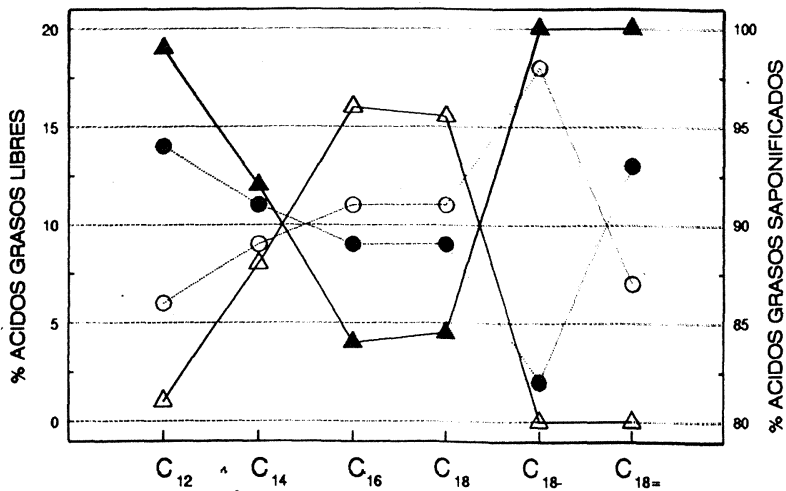

ACIDOS GRASOS ESTUDADOS

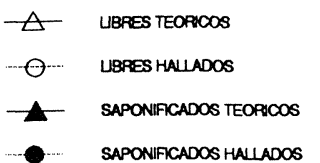

Figura 3

Comparación entre los ácidos grasos teóricos y los hallados en la crema III a temperatura ambiente.

libre posee un perfil análogo al del jabón antes de ser sobreengrasado. Es evidente que en el caso de las cremas de afeitar, con su contenido en agua considerable, $y$ debido a la presencia de dos cationes ( $\mathrm{Na}-\mathrm{K}$ ) con distinta reactividad frente a los ácidos grasos de diferentes longitudes de cadena, este fenómeno de intercambio ha de verse muy favorecido, quizás debido a la formación de jabones ácidos.

Desde el punto de vista práctico este hecho puede 


\section{DISTRIBUCION DE ACIDOS GRASOS LIBRES Y SAPONIFICADOS} CREMA I A TEMPERATURA AMBIENTE

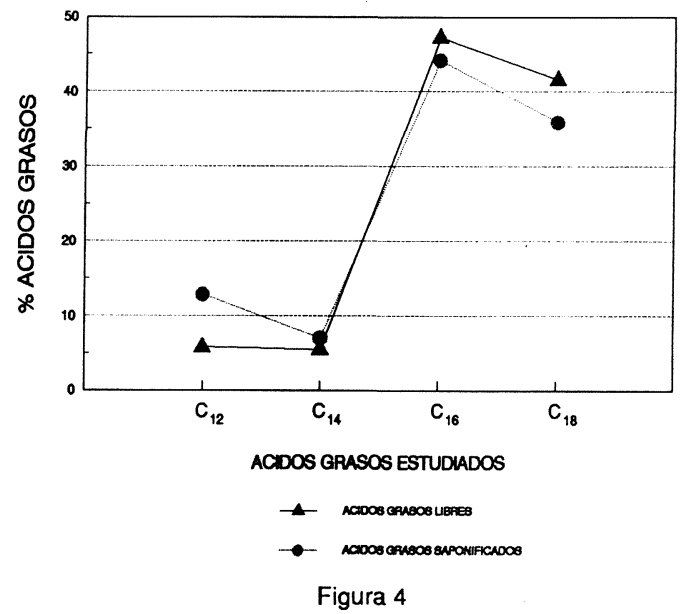

Se comprueba que la distribución de los ácidos grasos libres es similar a la de los ácidos grasos saponificados.

DISTRIBUCION DE ACIDOS GRASOS LIBRES Y SAPONIFICADOS CREMA II A TEMPERATURA AMBIENTE

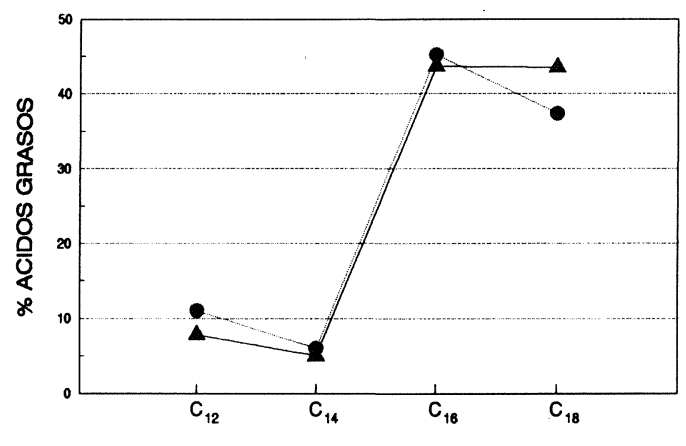

ACIDOS GRASOS ESTUDUADOS

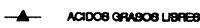

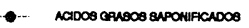

Figura 5

Se comprueba que la distribución de los ácidos grasos libres es similar a la de los ácidos grasos saponificados.

DISTRIBUCION DE ACIDOS GRASOS LIBRES Y SAPONIFICADOS CREMA III A TEMPERATURA AMBIENTE

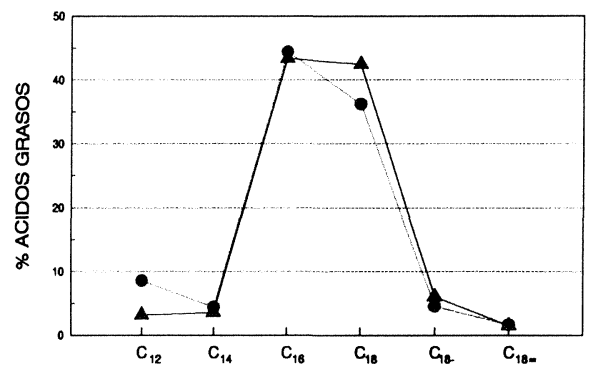

ACIDOS GRASOS ESTUDIADOS

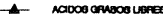

$$
\text { Figura } 6
$$

Se comprueba que la distribución de los ácidos grasos libres es similar a la de los ácidos grasos saponificados. repercutir, por una parte, en el comportamiento de la crema frente a la temperatura, pues al existir como ácidos grasos libres mayor cantidad de cadenas cortas o insaturadas que se solubilizan en la estructura micelar formando micelas mixtas, la crema será más fluída y se verá menos afectada por la temperatura, sobre todo si se trata de ácidos no saturados líquidos a la temperatura de utilización de la misma.

Por otra parte, al existir ácidos grasos libres insaturados, susceptibles de enranciamiento, es aconsejable protegerlos con antioxidantes.

\section{CONCLUSIONES}

1. Se ha comprobado y cuantificado el fenómeno del desplazamiento del sobreengrasante en jabones blandos (cremas de afeitar).

2. Se ha encontrado que la composición final del insaponificado es similar en todas las cremas estudiadas e independiente de la cantidad de sobreengrasante añadido.

3. Se ha demostrado que para conocer la naturaleza del sobreengrasante de un jabón blando no es suficiente con realizar un análisis cromatográfico del insaponificado, ya que éste puede conducir a conclusiones equivocadas.

4. Se ha comprobado que,al menos en las muestras estudiadas, los ácidos grasos de cadena más corta, $\mathrm{C}_{1}$ y $\mathrm{C}_{14}$ son desplazados preferentemente del jabón e intercambiados por ácidos grasos de cadenas más largas, $C_{16}$ y $C_{18}$. En el caso de los ácidos grasos insaturados, se observa que éstos también son desplazados preferentemente del jabón e intercambiados por ácidos grasos saturados como palmítico y esteárico.

\section{BIBLIOGRAFIA}

1. Davidson, J., Better, E.J. y Davidson, A. -»Soap manufacture".- Vol. 1.- Ed. Interscience. Inc.- New York and London, 1953.

2. Norma ISO 1067.

3. Metcalfe and Schmidt.- Anal. Chem. 31 (1959) 307-311.

4. Sheppard, A.J. y Iverson, J.L.- J. Chem. Soc. 13 (1975) 448-452.

5. Wolff, J.P. - "Controle analytique des propriétés des savons de toilette".- Rev. Fr. Corps Gras 17 (1970) 659-665.

6. Tomlison, K. -"Savon surgraissé».- Rev. Fr. Corps Gras 20 (1973) 697 700.

7. Phipps, H.E.- Monitor June (1974) 255-259.

8. Official Methods of Analysis.- $15^{\text {th }}$ Edition.- AOAC, Vol. 2. methods 969.63 (1990) 963.

9. Official Methods of Analysis.- $15^{\text {th }}$ Edition.- AOAC, Vol. 2. methods 963.22 (1990) 964.

10. Guiochon, G. y Guillemin, C.L. -"Quantitative gas chromatography".J. Chrom. Lib. 42 (1988) 653.

(Recibido: Junio 1991)

Se continuará este trabajo utilizando otros disolventes extractores. 